Abstract

\title{
Unique Features of Immunity within the Immunoglobulin Heavy Chain Locus of Egyptian Rousette Bats ${ }^{\dagger}$
}

\author{
Maggie L. Bartlett 1,2,*, Peter A. Larson 2, Mariano Sanchez-Lockhart ${ }^{2}$ and Gustavo Palacios ${ }^{2}$ \\ 1 University of Nebraska Medical Center, Department of Immunology, Pathology and Infectious Diseases, \\ Omaha, NE 68182, USA \\ 2 United States Army Medical Research Institute of Infectious Diseases, Center for Genome Sciences, \\ Frederick, MD 21702, USA; peter.a.larson.ctr@mail.mil (P.A.L.); m.sanchez-lockhart@unmc.edu or \\ msanchezlockhart.ctr@mail.mil (M.S.-L.); gustavo.f.palacios.civ@mail.mil (G.P.) \\ * Correspondence: mbartl13@jh.edu or maggie.bartlett@unmc.edu \\ † Presented at Viruses 2020-Novel Concepts in Virology, Barcelona, Spain, 5-7 February 2020. \\ Published: 11 August 2020
}

\begin{abstract}
Marburg virus (MARV) presents with a hemorrhagic fever in primates but asymptomatically in its known reservoir, the Egyptian rousette bat (Rousettus aegyptiacus, ERB). Understanding the biological mechanisms that explain these differential outcomes could be used to develop efficient therapeutics against MARV disease in humans. Since one of the antiviral mechanisms to control viruses is the humoral response, we hypothesize that the B cell repertoire is unique to primates and contributes to the ERB's ability to overcome MARV infection. Immunoglobulin (Ig) heavy and light chains undergo DNA rearrangement to generate a diverse repertoire. To be able to study $\mathrm{B}$ cell rearrangement, the accurate annotation of the Ig heavy chain $(I G H)$ locus is needed. We implemented three complementary strategies to describe and annotate the IGH locus of ERBs. First, we identified and annotated genes at the IGH locus, utilizing the previously described genome and transcriptome of the ERB our group created in collaboration with the CDC and the University of Boston. Second, we sequenced the specific IgM transcriptome of B cells from ERB peripheral blood mononuclear cells (PBMCs), to confirm or identify new IGH germline genes. Third, we generated bacterial artificial chromosome (BAC) libraries to confirm and improve the layout of the IGH locus. We were able to resolve misassemblies of these regions and identify multiple gene expansions unique to ERBs that may contribute to their ability to generate $B$ cell diversity and control infections. We found an expansion of genes associated with protection from various viruses in humans, differential expression of ERB isotypes across tissues, and two functional $I g E$ genes.
\end{abstract}

Keywords: Egyptian rousette bat; immunoglobulin heavy chain locus

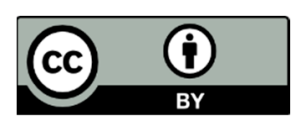

(C) 2020 by the authors. Licensee MDPI, Basel, Switzerland. This article is an open access article distributed under the terms and conditions of the Creative Commons Attribution (CC BY) license (http://creativecommons.org/licenses/by/4.0/). 